\title{
Eruptive Xanthoma with Acute Pancreatitis in a Patient with Hypertriglyceridemia and Diabetes
}

\author{
Tomoko Inoue-Nishimoto $^{1}$, Takaaki Hanafusa ${ }^{1,3}$, Ayaki Hirohata ${ }^{1}$, Eriko Kiyohara-Mabuchi ${ }^{1}$, \\ Naho Mizoguchi ${ }^{1}$, Kohei Matsumoto ${ }^{1}$, Sachiko Sakamoto $^{1}$, Masako Zenibayashi ${ }^{2}$, Satoshi Miyata ${ }^{2}$, \\ Ryuta Ikegami $^{1}$
}

Departments of ${ }^{1}$ Dermatology and ${ }^{2}$ Medicine, Japan Community Healthcare Organization Osaka Hospital, Osaka, ${ }^{3}$ Department of Dermatology, Graduate School of Medical and Dental Sciences, Tokyo Medical and Dental University, Tokyo, Japan

\begin{abstract}
Dear Editor:
A 41-year-old Japanese man presented in October 2013 with a 2-week history of pruritic, erythematous papules. He had diabetes mellitus, hyperlipidemia, and hypertriglyceridemia, but had stopped treatment 5 years prior to this visit. He also suffered from acute pancreatitis, and was admitted to the Department of Medicine, Japan Community Healthcare Organization Osaka Hospital, 1 day prior. Topical steroid ointment prescribed during a previous visit to another clinic was ineffective for the treatment of the papules. At the time of consultation, he presented with multiple, approximately 5-mm, pruritic, yellowish, erythematous papules on the trunk and extensor surfaces of the extremities (Fig. 1A). Dermoscopic examination of the papules clearly revealed granular yellowish characteristics (Fig. 1B). The laboratory findings (normal values are in parentheses) were: white blood cell count, $13.3 \times 10^{3}$ $\left(4.0 \times 10^{3} \sim 8.5 \times 10^{3}\right) / \mu$ l; neutrophils, $83.5 \%(38 \% \sim 58 \%)$; alkaline phosphatase, $369(115 \sim 359) \mathrm{U} / \mathrm{L} ; \mathrm{C}$ reactive protein, $5.91(<0.3) \mathrm{mg} / \mathrm{dl}$; hemoglobin A1c, $9.2 \%(4.6 \%$ $6.2 \%)$; amylase, 340 (40 130) IU/L; lipase, 960 (11 53) $\mathrm{U} / \mathrm{L}$; triglyceride, 6,784 (30 150) mg/dl; total cholesterol, 803 (130/219) mg/dl; high density lipoprotein cholesterol, 12 (42 74) mg/dl; low density lipoprotein cholesterol, 23 (70 140) $\mathrm{mg} / \mathrm{dl}$; remnant-like lipoprotein particle cholesterol, $84.4(<7.5) \mathrm{mg} / \mathrm{dl}$; apolipoprotein (Apo) A-I, 89 (119 155) mg/dl; Apo A-II, 12.6 (25.9 35.7) mg/dl; Apo B, 135 (73 109) mg/dl; Apo E, 11.0 (2.7 4.3) mg/dl;
\end{abstract}

Apo C-II, $16.5(1.8 \sim 4.6)$ mg/dl; Apo C-III, 28.3 (5.8 10.0) $\mathrm{mg} / \mathrm{dl}$. A skin biopsy sample obtained from papules on the waist revealed foamy cells that infiltrated the dermis. At higher magnification, foamy cells and extracellular lipids were present (Fig. 2). Therefore, we diagnosed the patient as having eruptive xanthoma with type $\mathrm{V}$ hyperlipoproteinemia according to Fredrickson's classification of hyperlipoproteinemias. We treated the acute pancreatitis with fasting, nafamostat mesilate, and ulinastatin, and the hypercholesterolemia with bezafibrate. The acute pancreatitis improved 16 days after admission. Serum triglyceride and total cholesterol values decreased to within normal range 1.5 months after the initiation of treatment. The eruptive xanthoma lesions rapidly resolved as the hyperlipidemia improved.

Hyperlipidemia is divided into six subgroups. Cutaneous xanthomatous lesions can occur in all six types of hyperlipidemia ${ }^{1}$. Cutaneous xanthomas can be divided into subgroups, including eruptive xanthomas, tuberous xanthomas, tendon xanthomas, xanthelasmata, and plane xanthomas $^{2}$. Eruptive xanthomas can be associated with hypertriglyceridemia (Type I, IV, or V hypercholesterolemia), particularly when diabetes is poorly controlled ${ }^{2,3}$. Eruptive xanthomas contain more triglycerides and fewer cholesteryl esters than other types of xanthomas, and lipids present in xanthomas are derived from circulating plasma lipoproteins. Triglyceride is mobilized more rapidly than cholesterol, so resolving eruptive xanthomas are rich in

\section{Received November 24, 2014, Revised March 22, 2015, Accepted for publication April 8, 2015}

Corresponding author: Takaaki Hanafusa, Department of Dermatology, Graduate School of Medical and Dental Sciences, Tokyo Medical and Dental University, Yushima 1-5-45, Bunkyo-ku, Tokyo 113-8519, Japan. Tel: 81-3-5803-5282, Fax: 81-3-5803-5289, E-mail: hanaderm@ tmd.ac.jp

This is an Open Access article distributed under the terms of the Creative Commons Attribution Non-Commercial License (http:// creativecommons.org/licenses/by-nc/4.0) which permits unrestricted non-commercial use, distribution, and reproduction in any medium, provided the original work is properly cited. 

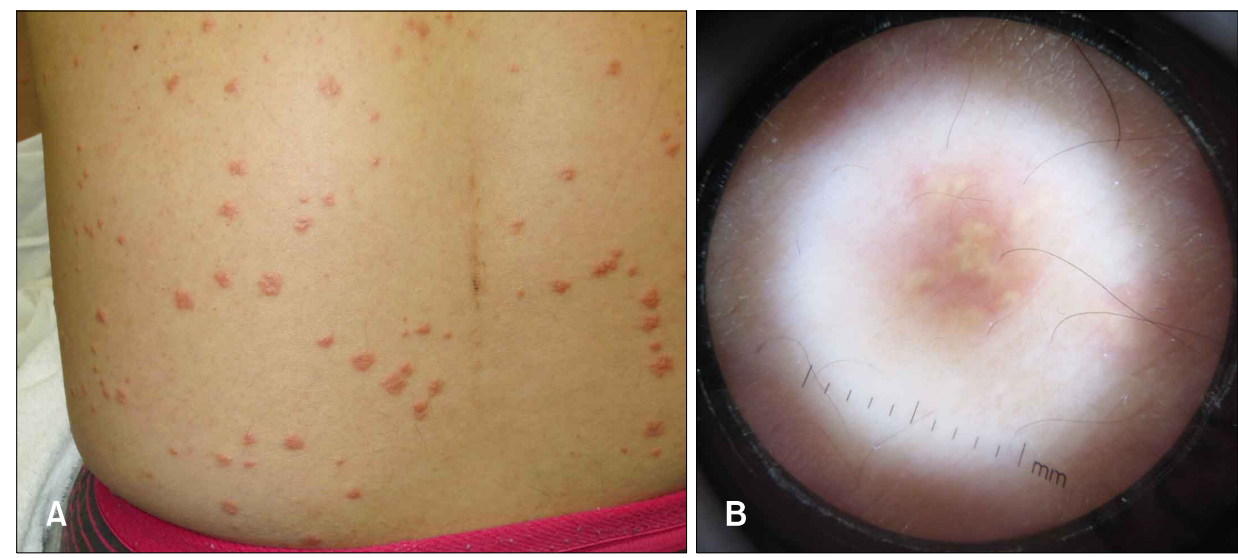

Fig. 1. (A) Clinical appearance and (B) dermoscopic appearance of multiple yellowish erythematous papules on the patient's back in October 2013.
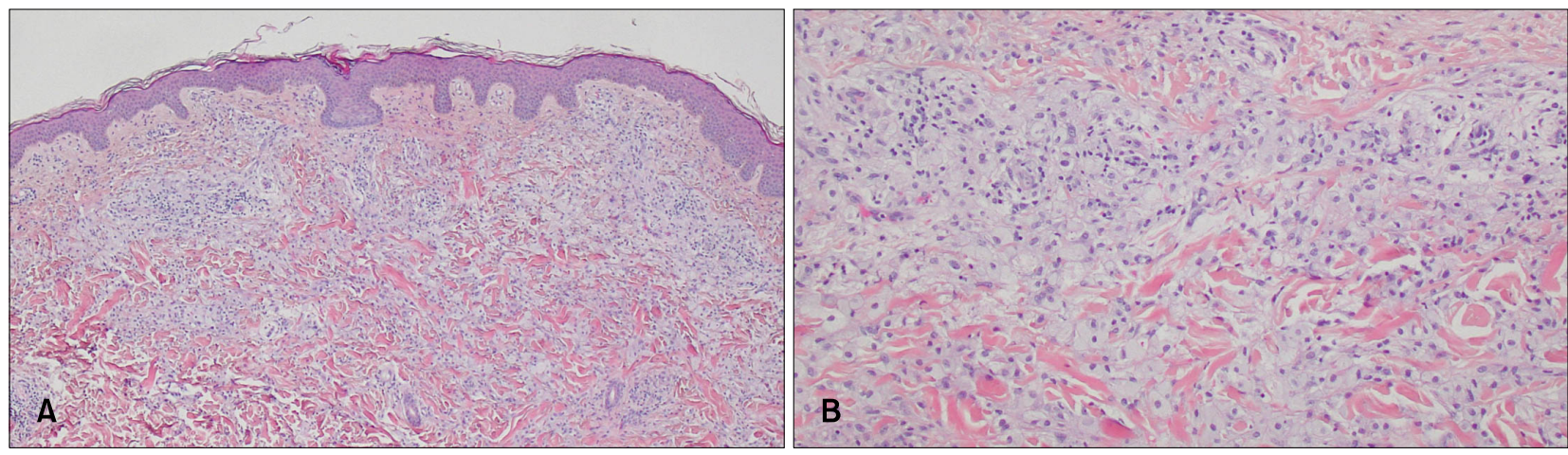

Fig. 2. Skin biopsy from the erythematous papules present on the patient's waist. (A) At lower magnification, numerous pale areas containing foamy cells infiltrate the dermis, especially the upper dermis and perivascular areas $(\mathrm{H} \& \mathrm{E}, \times 40)$. (B) At higher magnification, foamy cells with extracellular lipids are present $(\times 200)$.

cholesterol $^{4}$. Hypertriglyceridemia also increases the risk of acute pancreatitis, and the risk of eruptive xanthoma and pancreatitis increases when the serum triglyceride concentration reaches the thousands ${ }^{5}$. Eruptive xanthomas manifested in this patient 2 weeks before his visit, followed by acute pancreatitis. Dermatologists should carefully consider the possibility of acute pancreatitis with hypertriglyceridemia in eruptive xanthoma patients.

\section{REFERENCES}

1. Beaumont JL, Carlson LA, Cooper GR, Fejfar Z, Fredrickson DS, Strasser T. Classification of hyperlipidaemias and hyper- lipoproteinaemias. Bull World Health Organ 1970;43:891-915.

2. Parker F. Xanthomas and hyperlipidemias. J Am Acad Dermatol 1985;13:1-30.

3. Ladizinski B, Lee KC. Eruptive xanthomas in a patient with severe hypertriglyceridemia and type 2 diabetes. CMAJ 2013;185:1600.

4. Parker F, Bagdade JD, Odland GF, Bierman EL. Evidence for the chylomicron origin of lipids accumulating in diabetic eruptive xanthomas: a correlative lipid biochemical, histochemical, and electron microscopic study. J Clin Invest 1970;49:2172-2187.

5. Makdsi F, Fall A. Acute pancreatitis with eruptive xanthomas. J Hosp Med 2010;5:115. 